\title{
Influence of the Geometric Parameters on the Deposition Mode in Spatial Atomic Layer Deposition: A Novel Approach to Area-Selective Deposition
}

\author{
César Masse de la Huerta ${ }^{\circledR}$, Viet Huong Nguyen, Jean-Marc Dedulle, Daniel Bellet, \\ Carmen Jiménez $(\mathbb{D}$ and David Muñoz-Rojas *(D) \\ Université Grenoble Alpes, CNRS, Grenoble INP, LMGP, 38000 Grenoble, France; \\ cesar.masse@grenoble-inp.fr (C.M.d.1.H.); viet-huong.nguyen@grenoble-inp.fr (V.H.N.); \\ jean-marc.dedulle@grenoble-inp.fr (J.-M.D.); daniel.bellet@grenoble-inp.fr (D.B.); \\ carmen.jimenez@grenoble-inp.fr (C.J.) \\ * Correspondence: david.munoz-rojas@grenoble-inp.fr; Tel.: +33-456-529-337
}

Received: 3 November 2018; Accepted: 19 December 2018; Published: 22 December 2018

\begin{abstract}
Within the materials deposition techniques, Spatial Atomic Layer Deposition (SALD) is gaining momentum since it is a high throughput and low-cost alternative to conventional atomic layer deposition (ALD). SALD relies on a physical separation (rather than temporal separation, as is the case in conventional ALD) of gas-diluted reactants over the surface of the substrate by a region containing an inert gas. Thus, fluid dynamics play a role in SALD since precursor intermixing must be avoided in order to have surface-limited reactions leading to ALD growth, as opposed to chemical vapor deposition growth (CVD). Fluid dynamics in SALD mainly depends on the geometry of the reactor and its components. To quantify and understand the parameters that may influence the deposition of films in SALD, the present contribution describes a Computational Fluid Dynamics simulation that was coupled, using Comsol Multiphysics ${ }^{\circledR}$, with concentration diffusion and temperature-based surface chemical reactions to evaluate how different parameters influence precursor spatial separation. In particular, we have used the simulation of a close-proximity SALD reactor based on an injector manifold head. We show the effect of certain parameters in our system on the efficiency of the gas separation. Our results show that the injector head-substrate distance (also called deposition gap) needs to be carefully adjusted to prevent precursor intermixing and thus CVD growth. We also demonstrate that hindered flow due to a non-efficient evacuation of the flows through the head leads to precursor intermixing. Finally, we show that precursor intermixing can be used to perform area-selective deposition.
\end{abstract}

Keywords: spatial atomic layer deposition (SALD); computational fluid dynamics; surface reaction; thin films; ALD deposition; CVD deposition; area-selective deposition

\section{Introduction}

Atomic layer deposition (ALD) is a material deposition process that allows for a homogeneous, conformal thin film deposition with a nanometric thickness control. ALD is a type of chemical vapor deposition (CVD) method characterized by self-limited surface reactions. In ALD, instead of allowing a simultaneous presence of the reactants as is the case in the conventional CVD processes, a sequential exposure of the substrate to different reactants is needed to perform a chemical reaction with the substrate surface. A typical ALD cycle includes, periodically in time, exposure to a precursor, a purging step, an oxidant, and a second purging step. Vacuum processing is generally used in ALD in order to accelerate the purge steps and due to the traditional use of ALD in the microelectronics 
industries [1]. ALD cycles are characterized by having a defined growth per cycle (GPC) that depends on the chemical properties of the precursor, the temperature of the surface, and the reactor geometry. To attain a certain thickness, a determined number of cycles is performed. A review of the origins of ALD and a recommended reading list can be found in Reference [2].

Spatial Atomic Layer Deposition (SALD) is a technique based on the same principles of conventional (also called temporal) ALD, whose popularity is growing among the materials research community due to the fast deposition rates it offers, ranging from 20 to $40 \mathrm{~nm} / \mathrm{min}$, and to the large-area deposition capabilities at atmospheric pressure, and even in the open air, thus making it very appealing for the industry [3,4]. In addition, it offers the possibility of area-selective deposition $[5,6]$, simplicity of installation, and allows depositing high-quality materials with a higher throughput than ALD.

In Spatial ALD, the main difference with respect to conventional ALD resides on a spatial separation of continuously injected reactants. Instead of defining each step by a time separation, and to achieve the same chemical half reactions that take place during the temporal ALD cycles, in SALD, precursors are injected continuously in different spatial regions of the reactor and the substrate is exposed alternately to the different flows, separating each subsequent exposure with an intermediate exposure to an inert gas, to purge the substrate of the half-reaction by-products, and/or excess of precursor. This spatially separated exposure of the substrate is equivalent to the temporal ALD cycles and achieves comparable materials properties when the materials deposited are not sensitive to the atmosphere [7]. SALD has been tested before by several groups to deposit a wide variety of functional oxides in a homogeneous and conformal manner, in many cases taking place at atmospheric pressure [8-10].

Numerous approaches have been explored to successfully generate the mentioned spatial regions needed, without intermixing the gaseous precursors in SALD [9-12]. Specifically, the approach used in our laboratory (a home-made system presented and explained in detail in [4]) is based on a patent published by Kodak [13] that led to the publication of scientific papers using the spatial separation ALD concept by the same group from 2008 [4]. The system relies on a deposition head with linear gas outlets that injects above the substrate surface a given flow and concentration of reactants within an inert carrier gas. The substrate is usually placed at a close distance $(50-200 \mu \mathrm{m})$ during deposition, henceforth referred to as deposition gap. Such technique is commonly known as "close-proximity approach" since a small deposition gap value is necessary to prevent precursor intermixing across the inert gas region, thus avoiding a CVD regime deposition, i.e. reaction of the precursors reaching the substrate surface.

The spatial attribute of SALD gives many advantages with respect to temporal ALD, but due to the small value for the gap deposition needed, and to the fact that SALD is generally based on a mechanical displacement of the substrate, to fully exploit its advantages, a high mechanical and geometrical precision of the system needs to be carefully used. Furthermore, since our SALD approach does not rely on a chamber to be filled with the gases but rather on a continuous gas flow directed towards the surface of the substrate, the flow of such gases needs to be optimized as well to control the deposition conditions and to improve the homogeneity of the deposited film. Full control over these parameters is thus needed to enable a fast, large-area deposition with SALD.

Controlling the deposition gap can improve the versatility of the SALD, allowing it to tune the properties of the deposited film. In a CVD-like regime, films can be deposited in a fast way, but compactness, homogeneity, and control of thickness may be sacrificed. In an ALD regime, surface reactions on the substrate are self-limited, yielding a slower deposition rate, but a high conformality, homogeneity, and a good control of thickness are obtained in return. For the CVD regime to occur, intermixing of reactants must take place, yielding reactions above the surface before the precursors can reach it and be physi/chemisorbed. In contrast, in the ALD regime, the reactants must be chemisorbed, and ideally, saturate the surface before introducing the second reactant that leads to a complete surface reaction, thus creating a monolayer of the product. This key difference can be tuned arbitrarily in 
close-proximity SALD systems in which the deposition gap can be mechanically changed and thus it may provide versatility to tune the regime even in the middle of a deposition process [14]. A schematic of our injection head can be observed in Figure 1a, where the arrows represent the outlets and exhausts of the injected gases. The black arrows represent the inert carrier gas (I) that serves the purpose of confining the reactants and avoid intermixing. The white arrows represent exhausts (E) to which the gases can flow after being injected towards the surface of the substrate. The colored arrows represent the outlets of gases that contain the reactants used to create the surface reaction: the oxidant precursor $(\mathrm{OP})$ in red and the ALD metal precursor (MP) in blue. Figure $1 \mathrm{~b}$ shows the equivalent geometry used to perform the simulations. It is the region of interest from the original SALD schematic and corresponds to the region surrounded by a red dotted line in Figure 1a. The bottom-most line would represent the surface of the substrate and this line will be used as the place where surface reactions take place.

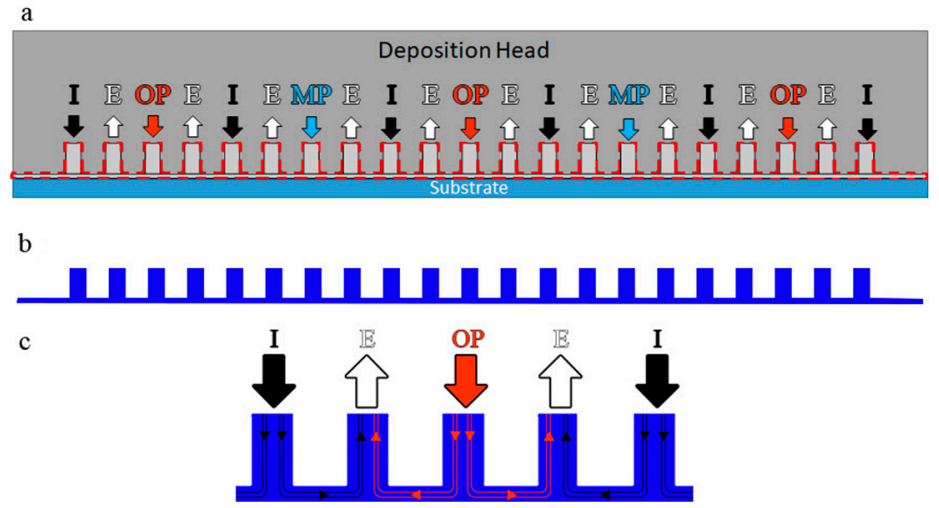

Figure 1. (a) Schematic of the cross-section of the deposition head in the spatial atomic layer deposition (SALD) system at the Laboratoire des Matériaux et du Génie Physique (LMGP). The gray section represents the deposition head on the system, while the blue section below represents a substrate. These two are separated by a space that corresponds to the deposition gap. (b) Equivalent geometry used for simulations used to compute all the phenomena in SALD regarding flows, concentrations, and reactions. (c) Close-up to the region of the OP, showing the expected flow lines and directions of the gaseous mixture of the SALD.

In this work, we have used computational fluid dynamics (CFD) simulations and coupled them with a surface reaction chemistry computation using Comsol Multiphysics ${ }^{\circledR} 5.3$ in order to model our homemade close-proximity SALD deposition system. Accordingly, the influence of different parameters of our SALD system on the intermixing of the gaseous precursors has been studied. A quantification of CVD or ALD deposition regime, which can affect the quality and homogeneity of the film deposited, has been calculated. The gas flows in the setup are studied for a controlled separation of species in the reactor, and adjusted to control the appearance of a CVD component in the deposition, as has indeed been reported in the past $[4,11]$. We show that the capability of controlling the deposition regime can be indeed used to have area-selective deposition with a close-proximity SALD. Finally, tolerances on the geometry and on the mechanical design of the system are presented as a guide towards a correct mechanical design of a versatile and reproducible SALD deposition system.

\section{Methods and Processes}

To calculate the influence of the deposition gap on the growth regime in our system, a Comsol Multiphysics ${ }^{\circledR}$ simulation, which couples CFD with concentrated species diffusion and with surface chemical reactions, was used. For this, an equivalent geometry that includes the gap and the outlets and exhausts of the deposition head was used to compute the gas flow to then couple it with the reactant concentration distribution in the flow, and ultimately, with the surface chemical reaction that happens on the substrate surface. A schematic representation of this equivalent geometry is shown 
in Figure 1b. Reference [15] presents a numerical study of a flow in a close-proximity system and concludes that, in the deposition gaps usually used in close-proximity SALD (typically 50-200 $\mu \mathrm{m}$ ), the Péclet number is low. This means that the transport of reactants is dominated by diffusion rather than by convection. We thus expect that the diffusion will play a key role in the behavior of our close-proximity system as well.

As said previously, this work simulates the gas flow that occurs with the gas outlets and the exhausts, as well as their influence on the distribution of reactant concentration on the substrate surface as a function of certain parameters of the system. This allows us to elucidate how such parameters influence on the existence of a CVD regime (i.e., precursor intermixing taking place). The simulation involved the study of the efficiency of the system to prevent regions where CVD could occur by calculating the conditions on which both reactants can be in contact prior to being adsorbed onto the substrate. The simulation has not considered a head/substrate relative movement during the deposition since the scope is only the efficiency of gas separation as a function of the different parameters studied.

Thus, the concentration of each reactant was computed at the immediate region above the surface of the substrate and the section in which both reactants are present was quantified (vide infra).

The SALD reactants consist of an ALD metal precursor and an oxidant. In the case of this work, Diethylzinc (DEZ) was used as the metal precursor, and water as the oxidant. To maintain the physical conditions as close as possible to the real conditions used, gas inputs of the injection head were taken as 300,450 , and $900 \mathrm{sccm}$ for the precursor (DEZ), oxidant $\left(\mathrm{H}_{2} \mathrm{O}\right)$, and nitrogen separation, respectively. These values are in accordance with what is commonly used when depositing zinc oxide $(\mathrm{ZnO})$ films with our system [7]. The diffusion coefficient of the reactants used was in the order of magnitude of $10^{-3} \mathrm{~m}^{2} \cdot \mathrm{s}^{-1}[16]$.

For the experimental validation of the simulations described here, $\mathrm{ZnO}$ was deposited using the SALD system at LMGP. A nitrogen flow carrying DEZ as precursor and water as oxidant to perform the surface reaction were used. A flow of pure nitrogen was used as the gas barrier between the reactants. The flows used were $300 \mathrm{sccm}$ for DEZ, $450 \mathrm{sccm}$ for water, and $900 \mathrm{sccm}$ for the nitrogen separation. The substrate was heated during deposition at $200^{\circ} \mathrm{C}$. The scanning speed of the substrate was $50 \mathrm{~mm} / \mathrm{s}$.

In order to simulate the reactions that happen during a SALD deposition, it is important to understand the simulation workflow. The ultimate objective is to study the surface reaction of species onto the substrate surface. For this, we first perform a zero-dimensional simulation of the CVD reaction, following the equation:

$$
\mathrm{DEZ}+\mathrm{H}_{2} \mathrm{O} \stackrel{R_{\mathrm{AB}}}{\rightarrow} \mathrm{CVD} \text { adsorbed film }
$$

which assumes that whenever DEZ and water molecules meet above the substrate they will react to form $\mathrm{ZnO}$. Equation (1) allows quantifying the amount of deposition obtained in a CVD regime.

Then, a CFD analysis of the flows in the deposition gap was performed and the results are presented in the geometry shown in Figure 1c. This computation yields the velocity and pressure of the flow at every point. Next, the velocity component of the CFD results was used to calculate a diffusion of concentrated species along the geometry. As a result, the presence of each reactant at any point of the geometry can be obtained. Finally, a surface concentration due to the CVD regime reaction is calculated using Equation (1) and the pressure obtained in the CFD computation. For such a surface reaction to happen, both reactants (in this case DEZ and $\mathrm{H}_{2} \mathrm{O}$ ) need to be present at a given time. Thus, the final surface reaction will yield the amount of CVD regime deposition.

\section{Results and Discussion}

\subsection{Evaluation of the Velocity Profile and Pressure in the Head-Substrate Gap}

Figure 2a shows the velocity profile obtained for a geometry in which the deposition gap was fixed at $150 \mu \mathrm{m}$, a value that is commonly used in real depositions and using the gas flow values previously mentioned. The profile shows the expected flow from the gas outlets to the exhausts, but it shows a 
non-zero value on the lateral outflow regions of the simulation, which would indicate that the exhaust at the injection head may not be as efficient as required. The simulation shows a maximum velocity of $\sim 2 \mathrm{~m} \cdot \mathrm{s}^{-1}$ at the narrowest point on the fluid's path from the inlet to the exhaust. The pressure profile is also shown in Figure $2 b$ and it presents a gradient of pressure from the corner of the gas outlets to the corner of the exhausts. For comparison, Reference [17] presents a similar study on the deposition gap of another close-proximity system and concludes that a $2 \mathrm{~mm}$ gap with a "low pumping" at the exhausts would achieve a good spatial separation of the reactants. While their system also relies on inlets, exhausts and a deposition gap, the geometry is not planar, as opposed to our system. It is also important to notice that their system works at a pressure lower than the atmospheric pressure, which prevents confinement of the gaseous flows, and that the geometry of the system may enhance diffusion processes, and therefore, enhance intermixing when working at close-proximity. In our case, the pressure achieved by the geometry (especially in the corners close to the exhaust) is helpful to induce a flow towards the exhaust, hence improving the convective flow of the reactants to the exhaust and reducing the diffusive flow. This in turn contributes to preventing precursor intermixing.

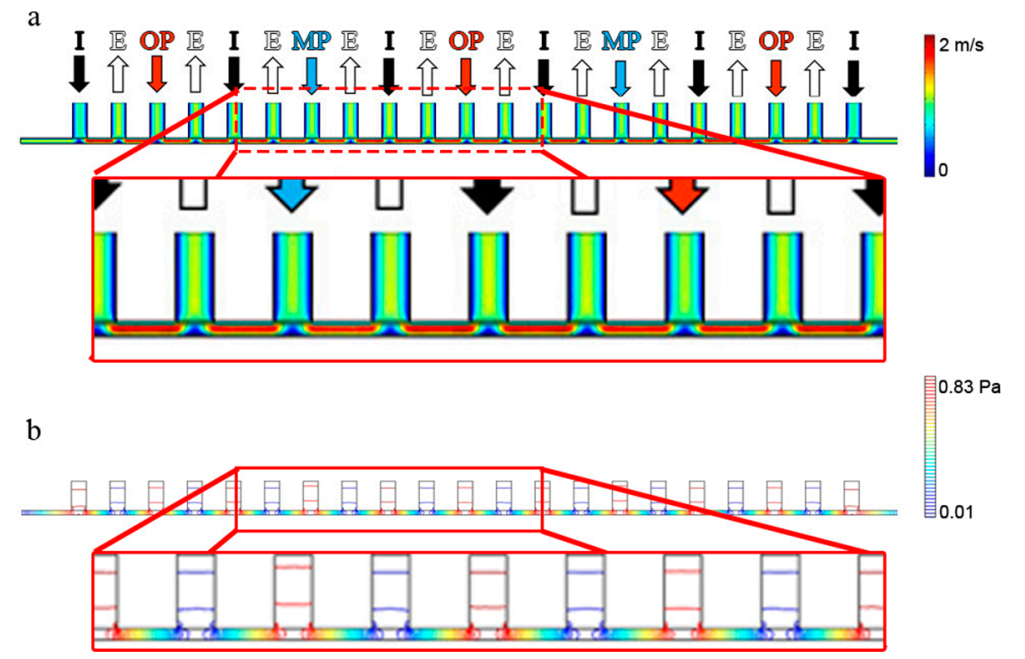

Figure 2. Computational Fluid Dynamics calculation made with Comsol Multiphysics ${ }^{\circledR}$ to represent the flow of the region of interest in the SALD geometry: (a) The velocity increases in the deposition gap, given the close proximity, and (b) the pressure increases under each of the outlets as it enters the deposition gap.

\subsection{Study of the Effect of the Head-Substrate Deposition Gap on the Deposition Mode}

Using the calculation of the velocity and the pressure obtained in the CFD computation, the concentration of each reactant was calculated along the gap, and more importantly, in the immediate region above the surface of the substrate for different deposition gaps. Figure 3 shows the concentration of the different reactants obtained along the length of the substrate $(30 \mathrm{~mm})$.

One can observe that, for a gap of $150 \mu \mathrm{m}$, the separation of reactants is well achieved (Figure 3a). In the concentration plot, the colors that represent the concentration of reactants are well separated along the geometry, with dark blue color (i.e., no precursor) below and next to the inert gas inlet channels, thus indicating a well-defined ALD regime. On the other hand, when the deposition gap is increased to $750 \mu \mathrm{m}$, the concentration is no longer well defined, as shown by the light blue color in the regions between each precursor. Thus, with this deposition gap, the deposition occurs in CVD regime (Figure $3 b$ ). In addition, to clearly compare different deposition gaps, first we plot the concentration of reactants in the carrier gases at the immediate region above the substrate, and then we quantified the amount of "overlap" between the plot of each precursor (indicated by the gray region in Figure 3). Again, a deposition gap of $150 \mu \mathrm{m}$ shows almost no overlap, whereas a deposition gap of $750 \mu \mathrm{m}$ clearly shows a much greater overlap. 

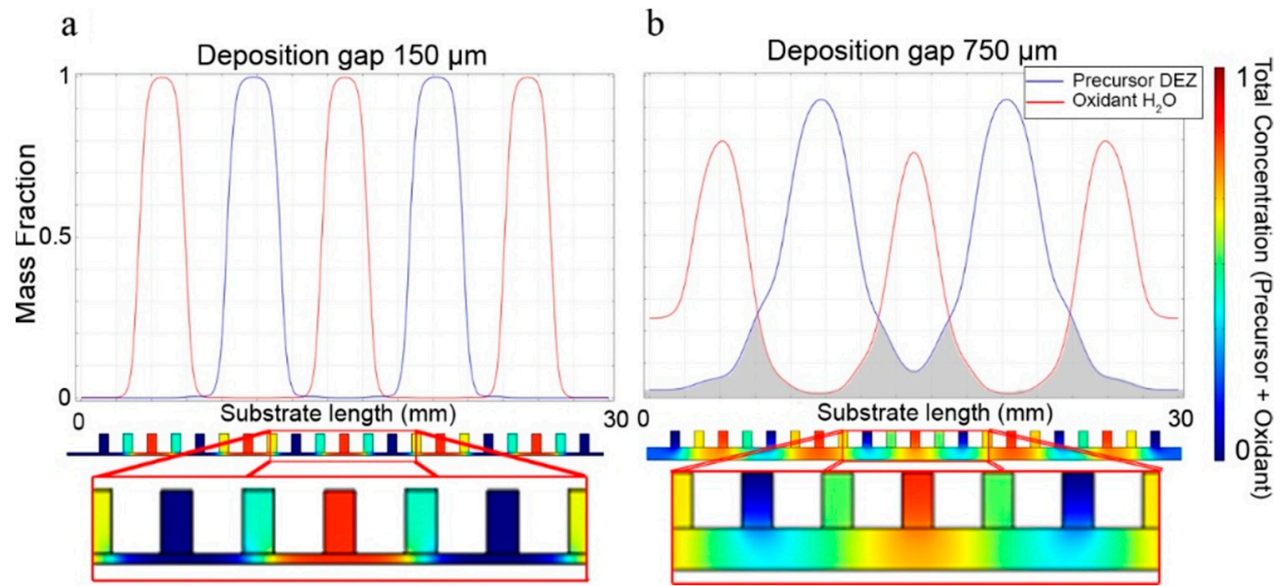

Figure 3. Concentration plot along the immediate region above the substrate for a deposition gap of (a) 150 and (b) $750 \mu \mathrm{m}$. Under each plot, a 2D plot along the whole geometry of the gap is shown, with a color code that corresponds to the concentration of reactants along the whole gap geometry.

Furthermore, in order to quantify the overlap for each deposition gap value, we calculated the ratio between the area under the curve of the overlap (where both reactants are present at the same time represented by the gray region in Figure 3) and the area under the curve of the sum of both concentrations, yielding a "percentage of overlap".

With this approach, further calculations of the percentage of overlap as a function of the deposition gap were made and are shown in Figure 4. This graph shows that, as the gap is increased, the overlap increases as well. Nevertheless, at a deposition gap value of around $750 \mu \mathrm{m}$ the gases mainly flow out through the sides of the head, rather than being confined on top of the substrate, and therefore there is a change in the slope of the curve and a lower overlap than it would be expected takes place.
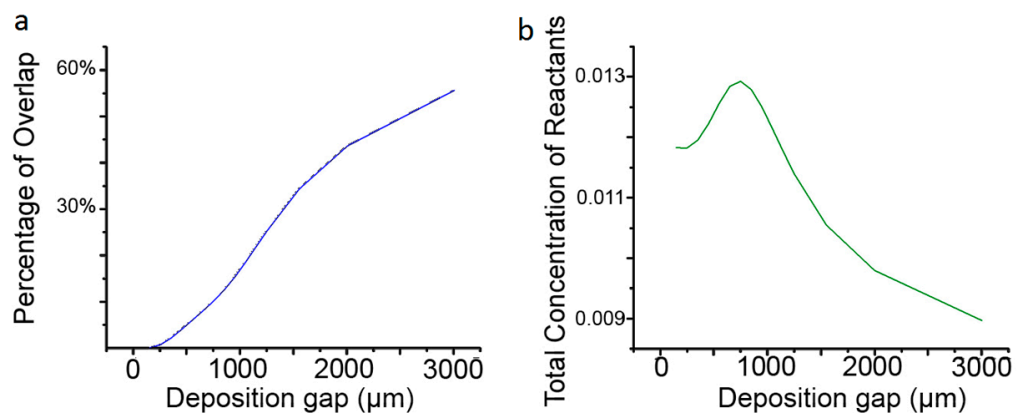

Figure 4. (a) The percentage of overlap, i.e., the percentage in which there exist both reactants at the same time, creating thus a CVD regime reaction on the surface of the substrate; (b) The total concentration of all the gases (both separated and overlapped) at the immediate area above the substrate vs. head-surface deposition gap.

Figure 4 also shows a plot of the total concentration of all reactants in the immediate region above the surface of the substrate, as measured by integrating the sum of the concentration of both reactants at the line of the substrate. The concentration reaches a maximum at $\sim 750 \mu \mathrm{m}$, which means that, at that deposition gap, the flow is preferentially directed towards the surface of the substrate, rather than at the lateral outflow regions. Nevertheless, the overlap percentage at that deposition gap is $\sim 13 \%$, which indicates the existence of a CVD component taking place. Logically, as the deposition gap increases, the flow tends to be directed to the lateral outflow regions rather than be captured at the injection head exhaust, making the extraction of the surplus of reactants difficult, leading to a release of chemicals to the atmosphere, which should, of course, be avoided. 
Study of the CVD Mode as a Consequence of Precursor Crosstalk

On a SALD reactor, reactions take place on the surface of the substrate, thus generating the desired films. Such reactions, in principle through chemisorption, take place as a consequence of the presence of a certain concentration of a reactant above the surface, and this depends on the pressure at each point, and of the substrate surface temperature.

In the case of an ALD deposition, the film deposition happens in two sequential half-reactions on the surface. Each half-reaction is self-limited to the surface sites available. In the case of SALD, if we assume a correct separation of the reactants, a static substrate, and a correct extraction of the reactant surplus, regardless of the time of injection of gases, the concentration of adsorbed reactant molecules in the surface should not be higher than the concentration of available sites. The surface coverage $(\theta)$ can be explained with the help of a Langmuir isotherm [18]:

$$
\theta=\frac{k_{a d s} P}{k_{d e s}+k_{a d s} P}=\frac{\text { (number of occupied sites) }}{\text { (total number of sites) }} ; 0 \leq \theta \leq 1
$$

where $k_{\text {ads }}$ and $k_{\text {des }}$ represent, respectively, the rate of adsorption/desorption of the reactant to/from the surface, and $P$ is the precursor partial pressure.

Furthermore, the reaction probability depends on the surface coverage, since, as more sites are occupied, the sticking probability $\beta$ of a reactant diminishes:

$$
\beta=\beta_{0}(1-\theta)
$$

where $\beta_{0}$ is the "bare reaction probability", given by the intrinsic properties of the reactant. Equation (3) is taken from [19], which also mentions that the saturation time of the reactant (our ALD precursor in this case) is inversely proportional to the precursor pressure and to $\beta_{0}$.

In contrast to an ALD deposition, in a CVD deposition, the reactants are present in the gas simultaneously, which will induce both a surface chemical reaction, due to both chemisorption and thermal activation of the reaction, and at a lower rate, at the gas phase. In the substrate surface, this CVD reaction will induce a competition among the reactants for the available surface sites. The reactants may react to be chemisorbed or may be desorbed from the surface. To describe this phenomenon, the Langmuir-Hinshelwood reaction rate equation can be used [18]:

$$
R_{\mathrm{AB}}=\frac{\mathrm{k}_{\mathrm{react}} \mathrm{K}_{\mathrm{A}} \mathrm{K}_{\mathrm{B}} P_{\mathrm{A}} P_{\mathrm{B}}}{\left(1+\mathrm{K}_{\mathrm{A}} P_{\mathrm{A}}+\mathrm{K}_{\mathrm{B}} P_{\mathrm{B}}\right)}
$$

where $\mathrm{k}_{\text {react, }}, \mathrm{K}_{\mathrm{A}}$, and $\mathrm{K}_{\mathrm{B}}$ are reaction constants corresponding to the whole reaction, and to reactant $\mathrm{A}$ and $\mathrm{B}$, respectively, and $P_{\mathrm{A}}$ and $P_{\mathrm{B}}$ are the partial pressures of each reactant. To express the partial pressure of each reactant in terms of its concentration, we can assume an ideal gas behavior and express them as:

$$
P_{\mathrm{A}}=\frac{n_{\mathrm{A}}}{n} P=x_{\mathrm{A}} P
$$

and

$$
P_{\mathrm{B}}=\frac{n_{\mathrm{B}}}{n} P=x_{\mathrm{B}} P
$$

where $n_{\mathrm{A}}$ and $n_{\mathrm{B}}$ are the numbers of moles of each reactant, $n$ is the total number of moles of the solution and $x_{\mathrm{A}}$ and $x_{\mathrm{B}}$ are the partial fractions of each reactant. Hence, substituting in Equation (4), we can obtain:

$$
R_{\mathrm{AB}}=\frac{\mathrm{k}_{\text {react }} \mathrm{K}_{\mathrm{A}} \mathrm{K}_{\mathrm{B}} x_{\mathrm{A}} x_{\mathrm{B}} P}{\left(\frac{1}{P}+\mathrm{K}_{\mathrm{A}} x_{\mathrm{A}}+\mathrm{K}_{\mathrm{B}} x_{\mathrm{B}}\right)}
$$


In Equation (7), the reaction rate of each reactant is considered, as well as a reaction rate of the reaction as a whole. This equation indicates that the reaction rate is not self-limited and will, therefore, continue as long as there is a non-zero concentration for both reactants. It also implies that if at any point the mass fraction of any of the reactants is zero, the reaction rate will also be zero and hence, no reaction would occur.

With this in mind, several time-dependent simulations were carried out in Comsol Multiphysics ${ }^{\circledR}$ to observe the appearance of a CVD deposition regime for different values of the deposition gap. The surface concentration obtained in such CVD regime is characterized by Equation (1) (Section 2), while the reaction rate $R_{\mathrm{AB}}$ comes from Equation (7). The pressure and the mass fraction of each reactant are calculated before the time-dependent surface chemistry reaction study, in the laminar flow study presented in Section 3.1, and in the concentration simulation of each reactant in the flow, respectively. As the CVD reaction is not self-limited, such time-dependent simulation was limited to $1 \mathrm{~s}$. The reaction rate used for the CVD surface reaction has a value of $1.5 \times 10^{-5} \mathrm{~mol} \cdot \mathrm{s} \cdot \mathrm{kg}^{-1} \cdot \mathrm{m}^{-1}$ [18].

Figure 5 shows the result of simulations of a time-dependent surface reaction. On top, a plot that corresponds to the amount of $\mathrm{ZnO}$ film deposited as a result of CVD taking place is shown. Under each plot, a 2D color plot of the CVD reaction rate can be seen. It is clear that the highest value of reaction rate $R_{\mathrm{AB}}$ would lead to a thicker, CVD regime deposition. Confirming previous simulations described above, as the deposition gap increases, the diffusion of reactants presents more "overlap" and hence, the reaction rate is higher, leading to a higher CVD component in the process, which in turn yields higher surface concentration as the gap is increased (Figure 5a).

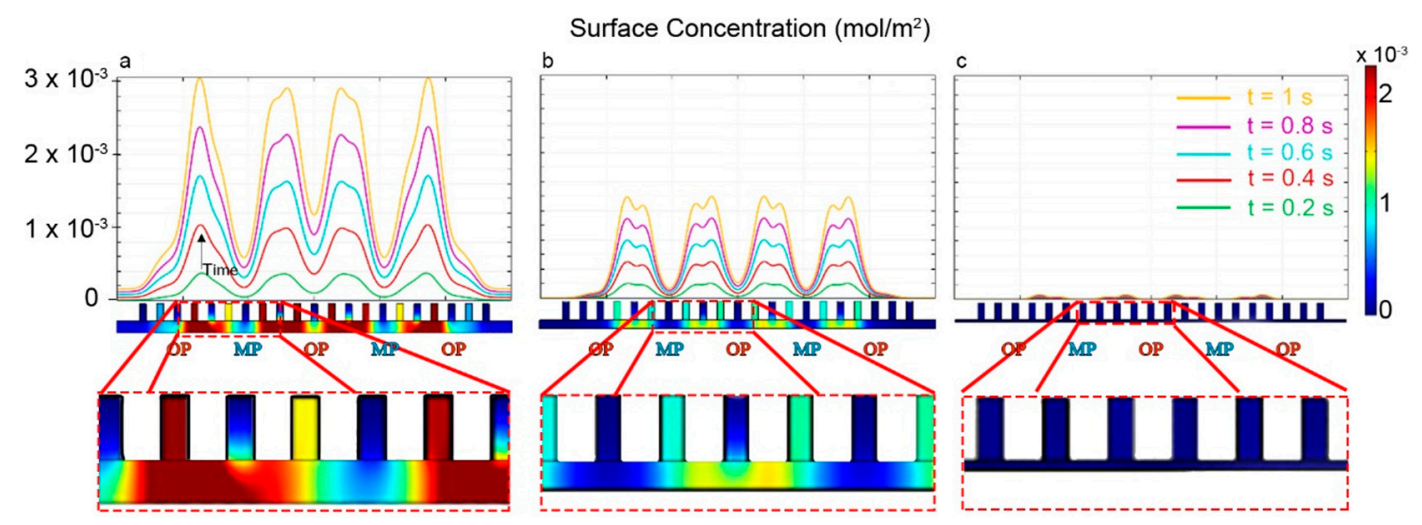

Figure 5. Results of the CVD surface reaction on the substrate calculated with a time-dependent multiphysics simulation. The plots shown correspond to the surface concentration that results from different gaps. Under each plot, a surface plot of the CVD reaction rate that corresponds to the plot directly above is shown, with OP and MP representing the outlets of the reactants; (a) deposition gap of $750 \mu \mathrm{m}$, (b) deposition gap of $425 \mu \mathrm{m}$, and (c) deposition gap of $150 \mu \mathrm{m}$.

To obtain evidence of the existence of the CVD and ALD regime with a simple change in gap, depositions of $\mathrm{ZnO}$ were made at different values of the gap. Figure 6 shows experimental results as evidence of the ability to modify the growth regime in our SALD system. Figure 6a presents the increase of growth rate as the gap value increases. The values for growth rate are in accordance with those reported for a self-limited (ALD) growth of $\mathrm{ZnO}$ [20-22]. The increase of the growth per cycle (GPC) with the gap value confirms the transition from an ALD regime (with small gap values) to a $\mathrm{CVD}$ regime (with higher gap values). Figure $6 \mathrm{~b}$ shows the $\mathrm{XRD}$ spectra of $\mathrm{ZnO}$ films grown with different gap values for the same number of cycles. The peaks correspond to those of crystalline $\mathrm{ZnO}$ and one can observe that, as the gap value increases, the intensity of the peaks increases as well, indicating that thicker films are obtained in the same deposition time as the gap is increased. 

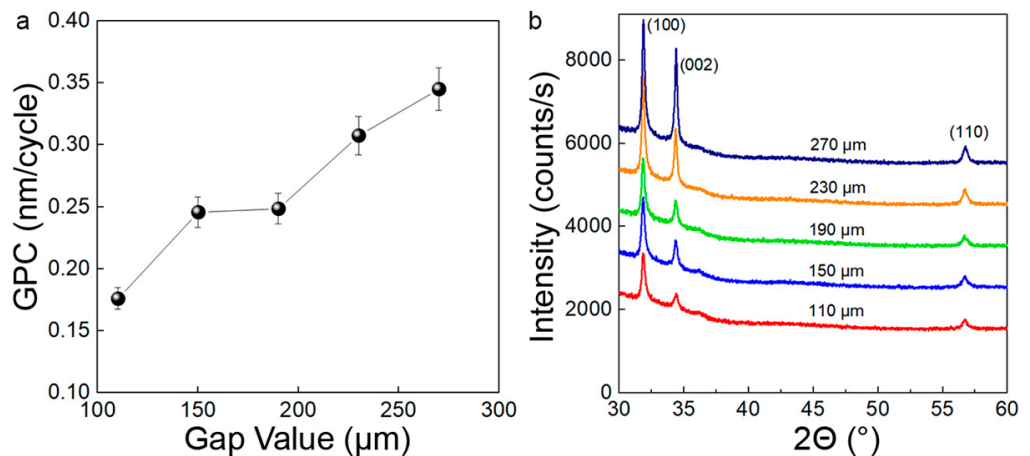

Figure 6. Experimental results for a deposition of $\mathrm{ZnO}$ using di-ethyl zinc (DEZ) and water as co-reactants. (a) Growth per cycle (GPC) evolution with different gap values; (b) X-ray diffraction patterns for $\mathrm{ZnO}$ films grown with different gap values, showing the crystalline peaks corresponding to wurzite $\mathrm{ZnO}$ (ICSD \#82028).

\subsection{Efficiency of the Deposition System Exhaust}

Using the same method described above, the exhaust efficiency was studied. In the geometry of our SALD deposition head, one assumes that all inputs will be directed towards the exhausts and that all surplus of reactant concentration is directed towards the exhausts (empty arrows in Figure 2). Nevertheless, to ensure this, the exhaust outlet must have the same outflow rate as the sum of all the inflow of gases (i.e., mass balance). Failure to achieve this, i.e., due to a bad design or to a partial or total blockage of the exhausts, may induce a CVD regime even with a small deposition gap. We define exhaust efficiency as the efficiency in which the incoming gaseous reactants and by-products are extracted from the reaction zone. A high exhaust efficiency may be achieved by a properly designed outlet/exhaust area ratio, or alternatively with, for example, properly chosen pumping in the exhausts. Here, we use a geometrical approach to such efficiency by calculating the outlet/exhaust area ratio, as explained below.

The result of simulations is shown for different exhaust efficiencies in Figure 7. We quantify an exhaust efficiency as the ratio between the total cross-section area of the exhausts and the total cross-section area of the gas outlets. An ideal ratio would be where the exhaust and the outlet have the same total area, in which case we consider an exhaust efficiency of $100 \%$. As the exhaust efficiency decreases, there is more diffusion of the reactant concentrations, even when the deposition gap is at a "close-proximity" of $150 \mu \mathrm{m}$. Interestingly, the diffusion of reactant concentrations has a slightly different behavior in this case than in the case of an increase of deposition gap. As the exhaust efficiency decreases, the CVD reaction rate appears to be more localized. This leads to a localized deposition in the CVD regime, and with time, four overlapped regions appear in localized points over the substrate.

To evaluate the behavior of the exhaust efficiency in real conditions, an experimental "static deposition" was performed to observe the behavior of the real SALD. The deposition head was placed at $150 \mu \mathrm{m}$ and all flows (precursor, oxidant and separation nitrogen) were injected as usual. The movement of the substrate was suppressed, and the pattern deposited was compared to simulations.

Figure 8 shows a "static deposition" using DEZ as metallic precursor and $\mathrm{H}_{2} \mathrm{O}$ as the oxidant. The deposited sample shows four well-defined stripes roughly at the location under the oxidant, similar to the plot of the exhaust efficiency of $4.5 \%$ shown in Figure 7 . The exhaust/outlet ratio measured on the physical exhausts and outlets of the geometry of our SALD system (measured as the ratio of the total cross-section area available for exhaust and the total cross-section area of gas outlets in our deposition head) is $10.1 \%$, which may explain the four lines pattern observed in our "static deposition". In order to improve the exhaust efficiency, one could envision either a change of the geometry of the deposition head or a forced exhaust using a pump. While it should be noted that adding a pump to the system will affect the fluid dynamics in the system, it may substantially change the deposition. 


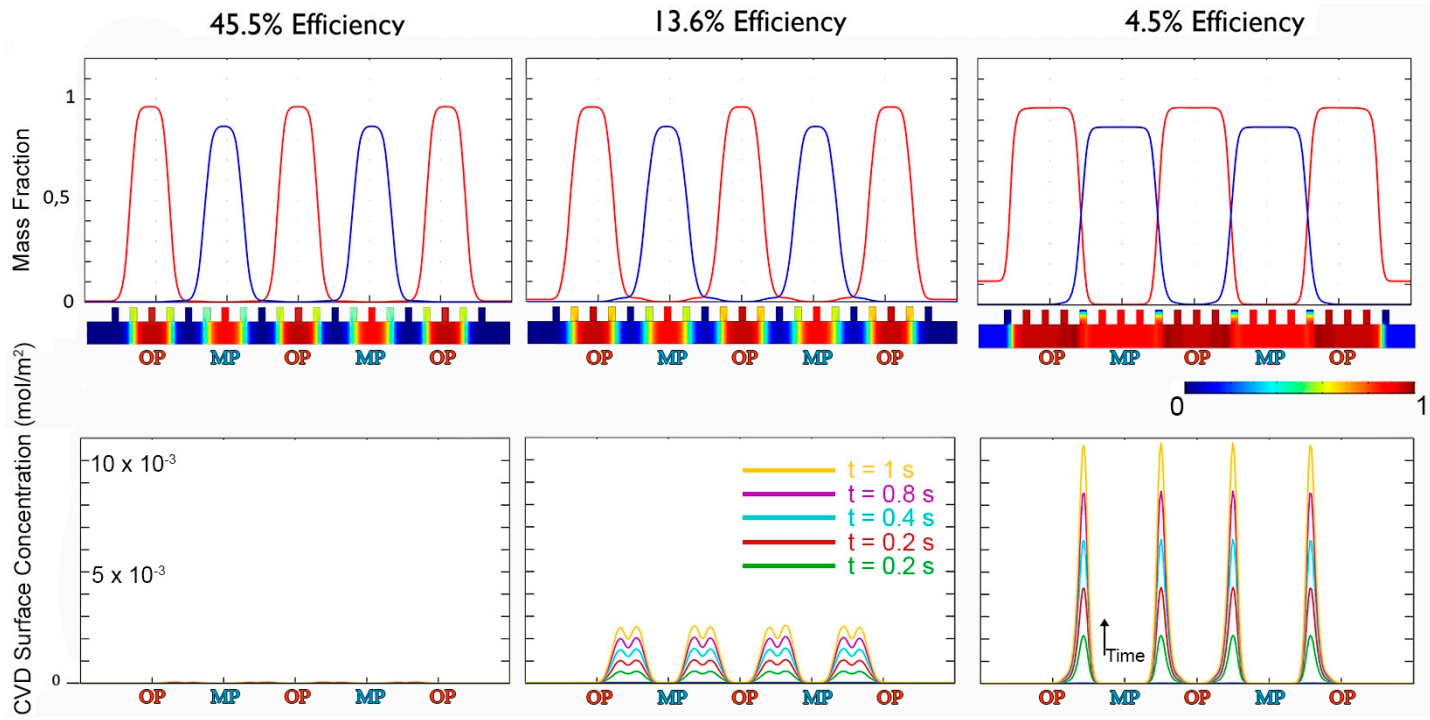

Figure 7. CVD regime deposition with different exhaust efficiencies. It can be seen that the exhaust efficiency has a drastic influence on the appearance of CVD regime: with an exhaust efficiency of $\sim 45 \%$, almost no appearance of CVD regime can be observed, at $\sim 13 \%$ CVD regime appears in some regions, and at $\sim 4 \%$ CVD regime is more pronounced and more localized.
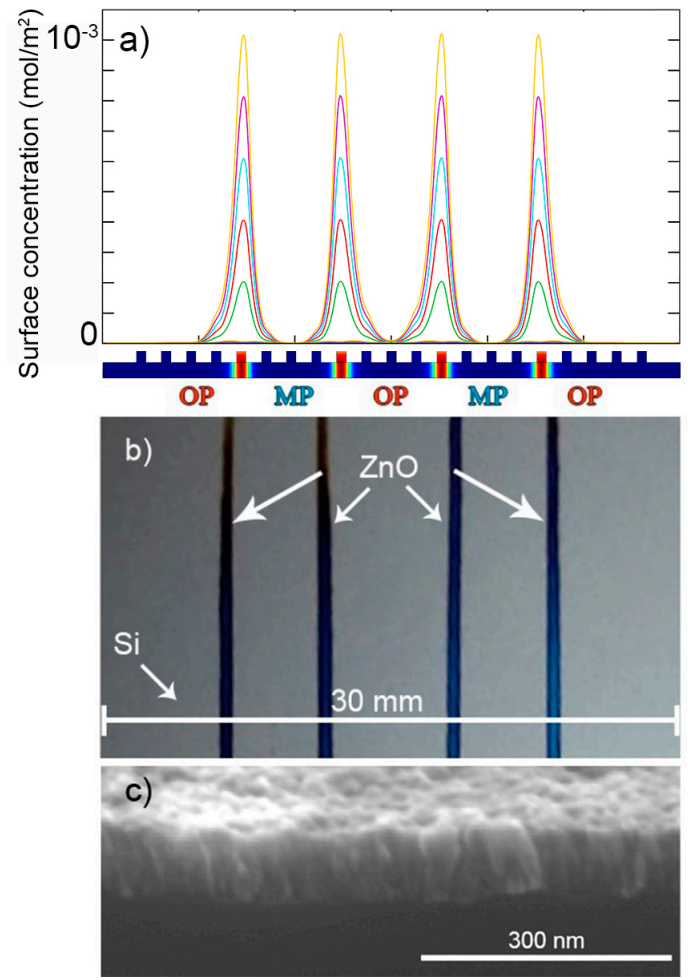

Figure 8. Simulation and experimental result of a static deposition experiment made with the SALD set-up at LMGP: (a) CVD deposition simulated for an exhaust efficiency of $\sim 4 \%$ shown previously in Figure 7, and (b) optical photography of the pattern obtained after performing an experimental "static deposition" on a Si substrate with DEZ and $\mathrm{H}_{2} \mathrm{O}$; (c) Scanning electron microscope (SEM) cross-section image showing a $\mathrm{ZnO}$ thickness of $\sim 75 \mathrm{~nm}$ for one of the lines in the pattern obtained after a $30 \mathrm{~s}$ long "static deposition". 


\subsection{CVD Regime Influenced by a Tilt in the Deposition Gap}

Finally, the CVD surface reaction was used to assess the influence of a tilt on either the substrate or the head. Since our SALD system uses a "close-proximity" approach where the deposition gap should be around $150 \mu \mathrm{m}$, a slight misalignment is expected to affect the distribution of gases (flows and pressures) and thus the deposition and homogeneity of the films. Figure 9 shows a CVD surface reaction of a tilted substrate by $0.3^{\circ}$.

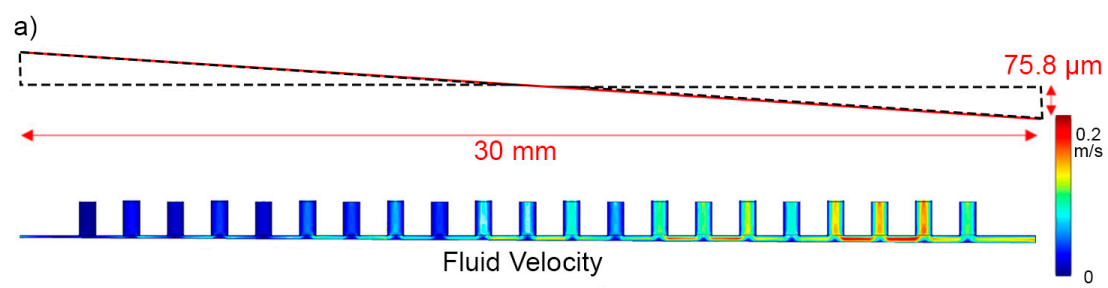

b)

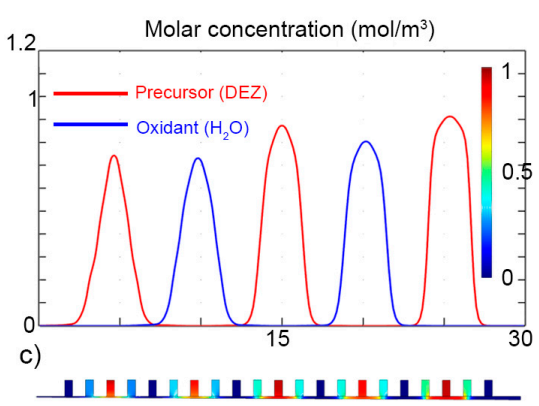

d)

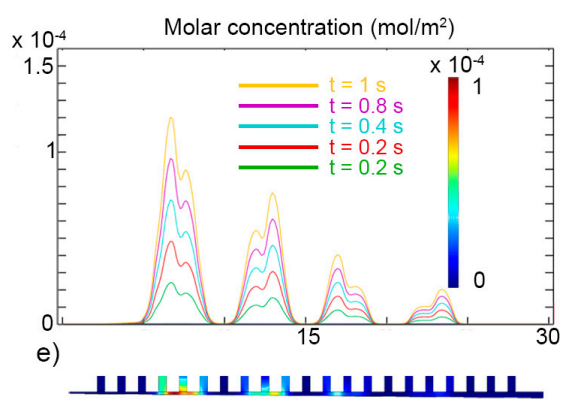

Figure 9. Simulation results for a tilt of $0.3^{\circ}$ : (a) Schematic of the rotation of the substrate with respect to the deposition head, about its center point, which leads to a difference of $\pm 75.8 \mu \mathrm{m}$ on each side of the substrate for a $30 \mathrm{~mm}$ length head. Below, the velocity of the flow is shown; (b) concentration of reactants above the surface; and (c) 2D concentration distribution in the gap. The concentration slightly favors the section with a higher deposition gap, (d) surface concentration of a film deposited as a consequence of the appearance of a CVD regime, and (e) 2D distribution of the CVD reaction rate.

As it can be seen in Figure 9, the influence of a small tilt can greatly affect the deposition. The $0.3^{\circ}$ tilt along the middle of the substrate for a $30 \mathrm{~mm}$ long deposition head causes a difference of $\sim 75.8 \mu \mathrm{m}$ at each extreme of the substrate, which changes the effective deposition gap, causing the deposition regime to change locally. Nevertheless, counter-intuitively to what was expected, the CVD reaction rate is higher in the section in which the deposition gap is narrower. This may be explained by the fact that, as the deposition gap decreases, the velocity, and hence the diffusion of the reactants, becomes higher, and the reactants have a higher chance of interacting between them creating a higher CVD reaction rate. As the gap widens, the velocity decreases and the flows are better separated by the nitrogen separation line and the exhausts, reducing the concentration of reactants and therefore the CVD reaction rate.

\section{Conclusions}

In the present work, Comsol Multiphysics ${ }^{\circledR}$ was used to study the influence of several geometrical parameters on the deposition regime when using a close-proximity SALD system. We confirmed that the deposition gap is crucial for the determination of an ALD or a CVD deposition regime and studied the influence of the "close-proximity" approach in the fluid dynamics. For a large deposition gap (i.e., larger than $\sim 500 \mu \mathrm{m}$ ), the separation of the flows is not achieved and intermixing of gas concentration appears. CVD regime can, therefore, be tuned simply by changing the value of the deposition gap. Hence, we present a simple and versatile way to tune the SALD deposition process by controlling simple parameters such as the head-substrate gap in the system. 
With respect to the CVD surface reaction, simulations were performed to study the CVD reaction rate at the surface of the substrate. Using the intermixing of concentrations calculated, two parameters were considered to determine the CVD reaction rate, and therefore, the CVD surface film formation, namely, the deposition gap and the exhaust efficiency. As expected, as the deposition gap is increased, the CVD reaction rate is also increased, and it gives place to a non-self-limited surface reaction on the substrate. In a $1 \mathrm{~s}$ simulation, a surface concentration of a film deposited in a CVD regime was plotted and a higher surface concentration was observed with a higher deposition gap. Concerning the exhaust efficiency, the ratio between the cross-section area of the exhausts with respect to the cross-section area of the outlets was investigated since this would define the exhaust efficiency for the surplus of precursor (and by-products of the surface reaction) present at the moment of deposition. Interestingly, with a ratio of $4.5 \%$, well localized intermixing of reactants was formed, leading to a four-stripes deposition pattern that is in accordance with the deposition obtained in a static experiment of a "static-deposition" performed. This would indicate that probably the exhaust efficiency of the current deposition head geometry must consider an exhaust/outlet ratio of less than $10 \%$, which is a value consistent with the real geometry of the deposition head used experimentally. Furthermore, with a precise mechanical design (of the gap and the inlets and exhausts of the deposition head) and with an optimized exhaust pumping, this behavior can be exploited as an approach for a selective area CVD or ALD deposition of materials.

Finally, the influence on a head-substrate tilt was studied. This is important since mis-alignment between the deposition head and the substrate is often difficult to avoid, and doing as such would require high-precision equipment, increasing the complexity of the instrumentation. Simulations show that a slight tilt of only $0.3^{\circ}$ is enough to change the deposition regime from one side of the substrate to the other. The simulations performed regarding the tilt influence have the aim to show the sensibility of the SALD to the positioning and portray the need of high precision on the system to precisely control the deposition.

The simulations performed in this study justify the need of high-precision and alignment of the geometry used. The parallelism of the system needs to be ensured as much as possible so that the deposition regime can be well controlled. In the current study, no movement of the substrate was studied but the same approach can be readily applied to evaluate the influence of the movement on the deposition regime, as well as on the precision requirements of the geometry.

As observed here, the geometry of the system is crucial to understand and control the deposition of thin films with SALD. With the correct geometry, area-selective deposition of films with properties comparable to ALD deposited films is possible, creating new advantages for SALD such as patterned mask-less film deposition, subsequent depositions for a multi-layer scheme, or a deposition with well optimized flows and concentrations to optimize the usage of ALD precursors.

Author Contributions: Conceptualization, C.M.d.1.H. and V.H.N.; Methodology, C.M.d.1.H. and V.H.N.; Validation, J.-M.D., C.J. and D.M.-R.; Investigation, C.M.d.1.H. and V.H.N.; Writing-Original Draft, C.M.H.; Writing-Review \& Editing, C.M.d.1.H., V.H.N., J.-M.D., D.B., C.J. and D.M.-R.; Supervision, D.B., C.J. and D.M.-R.; Funding Acquisition, D.M.-R.

Funding: This work was benefited from funding from the Consejo Nacional de Ciencia y Tecnología (CONACYT) from Mexico (No. 456558). The authors thank the "ARC Energies Auvergne-Rhône Alpes", for their economic support through a Ph.D. grant, and the Agence Nationale de Recherche (ANR, France) via the project DESPATCH (No. ANR-16-CE05-0021). This work benefited from the facilities and expertise of the OPE)N(RA characterization platform of FMNT (FR 2542, fmnt.fr) supported by CNRS, Grenoble INP, and UGA. DMR acknowledges funding through the Marie Curie Actions (FP7/ 2007-2013, Grant No. 631111). This project was financially supported by "Carnot Energies du Futur" (ALDASH project). This project has received funding from the European Union's Horizon 2020 FETOPEN-1-2016-2017 research and innovation programme under grant agreement 801464.

Acknowledgments: The author thanks Dominique De Barros for his support in the development of the SALD system at LMGP.

Conflicts of Interest: The authors declare no conflict of interest. 


\section{References}

1. Johnson, R.W.; Hultqvist, A.; Bent, S.F. A brief review of atomic layer deposition: From fundamentals to applications. Mater. Today 2014, 17, 236-246. [CrossRef]

2. Ahvenniemi, E.; Akbashev, A.R.; Ali, S.; Bechelany, M.; Berdova, M.; Boyadjiev, S.; Cameron, D.C.; Chen, R.; Chubarov, M.; Cremers, V.; et al. Review Article: Recommended reading list of early publications on atomic layer deposition-Outcome of the "Virtual Project on the History of ALD". J. Vac. Sci. Technol. A 2017, 35, 010801. [CrossRef]

3. Muñoz-Rojas, D.; MacManus-Driscoll, J. Spatial atmospheric atomic layer deposition: A new laboratory and industrial tool for low-cost photovoltaics. Mater. Horiz. 2014, 1, 314-320. [CrossRef]

4. Muñoz-Rojas, D.; Nguyen, V.H.; de la Huerta, C.M.; Aghazadehchors, S.; Jiménez, C.; Bellet, D. Spatial atomic layer deposition (SALD), an emerging tool for energy materials. Application to new-generation photovoltaic devices and transparent conductive materials. Comptes Rendus Phys. 2017, 18, 391-400. [CrossRef]

5. Levy, D.H.; Freeman, D.; Nelson, S.F.; Cowdery-Corvan, P.J.; Irving, L.M. Stable ZnO thin film transistors by fast open air atomic layer deposition. Appl. Phys. Lett. 2008, 92, 192101. [CrossRef]

6. Nelson, S.F.; Ellinger, C.R.; Levy, D.H. Improving yield and performance in ZnO thin-film transistors made using selective area deposition. ACS Appl. Mater. Interfaces 2015, 7, 2754-2759. [CrossRef] [PubMed]

7. Nguyen, V.H.; Gottlieb, U.; Valla, A.; Muñoz, D.; Bellet, D.; Muñoz-Rojas, D. Electron tunneling through grain boundaries in transparent conductive oxides and implications for electrical conductivity: The case of ZnO:Al thin films. Mater. Horiz. 2018, 5, 715-726. [CrossRef]

8. Choi, H.; Shin, S.; Jeon, H.; Choi, Y.; Kim, J.; Kim, S.; Chung, S.C.; Oh, K. Fast spatial atomic layer deposition of $\mathrm{Al}_{2} \mathrm{O}_{3}$ at low temperature $\left(<100{ }^{\circ} \mathrm{C}\right)$ as a gas permeation barrier for flexible organic light-emitting diode displays. J. Vac. Sci. Technol. A 2016, 34, 01A121. [CrossRef]

9. Poodt, P.; Lankhorst, A.; Roozeboom, F.; Spee, K.; Maas, D.; Vermeer, A. High-speed spatial atomic-layer deposition of aluminum oxide layers for solar cell passivation. Adv. Mater. 2010, 22, 3564-3567. [CrossRef] [PubMed]

10. Franke, S.; Baumkötter, M.; Monka, C.; Raabe, S.; Caspary, R.; Johannes, H.H.; Kowalsky, W.; Beck, S.; Pucci, A.; Gargouri, H. Alumina films as gas barrier layers grown by spatial atomic layer deposition with trimethylaluminum and different oxygen sources. J. Vac. Sci. Technol. A 2016, 35, 01B117. [CrossRef]

11. Muñoz-Rojas, D.; Jordan, M.; Yeoh, C.; Marin, A.T.; Kursumovic, A.; Dunlop, L.; Iza, D.C.; Chen, A.; Wang, H.; MacManus-driscoll, J.L. Growth of $\sim 5 \mathrm{~cm}^{2} \mathrm{~V}^{-1} \mathrm{~s}^{-1}$ mobility, p-type Copper $(\mathrm{I})$ oxide $(\mathrm{Cu} 2 \mathrm{O})$ films by fast atmospheric atomic layer deposition (AALD) at $225^{\circ} \mathrm{C}$ and below. AIP Adv. 2012, 2, 042179. [CrossRef]

12. Poodt, P.; Knaapen, R.; Illiberi, A.; Roozeboom, F.; van Asten, A. Low temperature and roll-to-roll spatial atomic layer deposition for flexible electronics. J. Vac. Sci. Technol. A 2012, 30, 01A142. [CrossRef]

13. Levy, D.H. Process for Atomic Layer Deposition. US Patent 7,413,982 B2, 19 August 2008.

14. Hoye, R.L.; Muñoz-Rojas, D.; Musselman, K.P.; Vaynzof, Y.; MacManus-Driscoll, J.L. Synthesis and modeling of uniform complex metal oxides by close-proximity atmospheric pressure chemical vapor deposition. ACS Appl. Mater. Interfaces 2015, 7, 10684-10694. [CrossRef] [PubMed]

15. Deng, Z.; He, W.; Duan, C.; Chen, R.; Shan, B. Mechanistic modeling study on process optimization and precursor utilization with atmospheric spatial atomic layer deposition. J. Vac. Sci. Technol. A 2015, 34, $01 \mathrm{~A} 108$. [CrossRef]

16. Van Deelen, J.; Illiberi, A.; Kniknie, B.; Steijvers, H.; Lankhorst, A.; Simons, P. APCVD of ZnO:Al, insight and control by modeling. Surf. Coat. Technol. 2013, 230, 239-244. [CrossRef]

17. Pan, D.; Jen, T.C.; Yuan, C. Effects of gap size, temperature and pumping pressure on the fluid dynamics and chemical kinetics of in-line spatial atomic layer deposition of $\mathrm{Al}_{2} \mathrm{O}_{3}$. Int. J. Heat Mass Transf. 2016, 96, 189-198. [CrossRef]

18. Dobkin, D.M.; Zuraw, M.K. Principles of Chemical Vapor Deposition, 1st ed.; Springer Science \& Business Media: Dordrecht, The Netherlands, 2013.

19. Yanguas-Gil, A. Growth and Transport in Nanostructured Materials: Reactive Transport in PVD, CVD, and ALD; Springer International Publishing: Cham, Switzerland, 2017.

20. Lim, J.; Lee, C. Effects of substrate temperature on the microstructure and photoluminescence properties of ZnO thin films prepared by atomic layer deposition. Thin Solid Films 2007, 515, 3335-3338. [CrossRef] 
21. Pal, D.; Mathur, A.; Singh, A.; Singhal, J.; Chattopadhyay, S. Photoluminescence of atomic layer deposition grown ZnO nanostructures. Mater. Today Proc. 2018, 5, 9965-9971. [CrossRef]

22. Rauwel, E.; Nilsen, O.; Rauwel, P.; Walmsley, J.C.; Frogner, H.B.; Rytter, E.; Fjellvåg, H. Oxide coating of alumina nanoporous structure using ALD to produce highly porous spinel. Chem. Vap. Depos. 2012, 18, 315-325. [CrossRef] 